Magdalena Zurawek ${ }^{1}$, Agnieszka Dzikiewicz-Krawczyk ${ }^{1}$, Katarzyna Izykowska', Iwona Ziolkowska-Suchanek1, Bogda Skowronska², Maria Czainska³ ${ }^{3}$, Marta Kazimierska1, Marta Podralska1, Piotr Fichna², Grzegorz Krzysztof Przybylski ${ }^{1}$, Jerzy Nowak ${ }^{1}$, Marta Fichna1, 4 , Natalia Rozwadowska ${ }^{1}$

${ }^{1}$ Institute of Human Genetics, Polish Academy of Sciences, Poznan, Poland

${ }^{2}$ Department of Paediatric Diabetes and Obesity, Poznan University of Medical Sciences, Poznan, Poland

${ }^{3}$ Family Physician Clinic, Murowana Goslina, Poland

${ }^{4}$ Department of Endocrinology and Metabolism, Poznan University of Medical Sciences, Poznan, Poland

\title{
Overexpression of miR-652-5p in new onset type 1 diabetes
}

\section{ABSTRACT}

Introduction. MicroRNAs (miRNAs) are small noncoding RNA regulating gene expression at the posttranscriptional level. miRNAs have emerged as an important regulators of central and peripheral immune tolerance, therefore study the RNA molecules in the context of type 1 diabetes (T1D) pathogenesis is an important issue. The aim of this study was to investigate miR-652-5p expression level in the new onset T1D and an impact on ADAR and MARCH5, potential target genes.

Material and methods. The miR-652-5p expression was investigated in the peripheral blood mononuclear cell of newly diagnosed T1D pediatric patients $(n=28)$ and age-matched controls $(n=28)$ by quantitative reverse-transcription polymerase chain reaction (qRT-PCR). miRNA targets were analyzed by luciferase reporter assays.

Results. Expression analysis revealed upregulation of miR-652-5p in T1D group compared to non-diabetic controls $(p<0.05)$. Luciferase reporter assay did not indicated $A D A R$ and MARCH5 as miR-652-5p targets.

Address for correspondence:

dr n. med. Magdalena Zurawek

Instytut Genetyki Człowieka, Polska Akademia Nauk

ul. Strzeszyńska 32, 60-479 Poznań

Phone: (+ 48) 6165792 81, fax: (+ 48) 618233235

e-mail: magdalena.zurawek@igcz.poznan.pl

Clinical Diabetology 2018, 7, 4, 189-195

DOI: $10.5603 /$ DK.2018.0019

Received: 04.06.2018

Accepted: 22.07.2018
Conclusion. Our study revealed miR-652-5p as potential marker of new onset type 1 diabetes. (Clin Diabetol 2018; 7, 4: 189-195)

Key words: T1D, expression, miR-652-5p, ADAR, MARCH5

\section{Introduction}

Type 1 diabetes (T1D) is a common autoimmune disorder caused by the T-cell mediated destruction of the insulin-producing pancreatic beta cells. The pathogenesis of T1D is a consequence of complex interplay between genetic, epigenetic and environmental factors [1].

Linkage studies have indicated the HLA class II genes at 6p21 as the major T1D susceptibility locus [2]. The concordance rate for monozygotic twins with high-risk HLA genotypes DR3-DQ2 and DR4-DQ8 and family history of T1D is estimated to be $\sim 50 \%$ [3]. To date, over 50 non-HLA susceptibility gene markers have been identified, mostly related to innate and adaptive immune response (http://www.t1dbase.org). These include the cytotoxic T-lymphocyte-associated protein 4 gene (CTLA-4), the protein tyrosine phosphatase, non-receptor type 22 gene (PTPN22), the interleukin 2 receptor subunit alpha gene (IL2RA) and the interferoninduced with helicase $C$ domain 1 gene (IFIH1) [4-7]. Among the environmental risk factors: cow's milk, vitamin D, glycotoxin, intestinal gut microbiota and enteroviruses might play a potential role in pathogenesis and progression of T1D [8-11]. Furthermore, epigenetic 
Table 1. Clinical features of the studied cohorts of patients with type 1 diabetes

\begin{tabular}{|c|c|c|c|}
\hline & $\begin{array}{c}\text { T1D } \\
\mathrm{n}=28(\%)\end{array}$ & $\begin{array}{c}\text { Severe } \\
\text { T1D onset } \\
n=12(\%)\end{array}$ & $\begin{array}{c}\text { Mild } \\
\text { T1D onset } \\
n=16(\%)\end{array}$ \\
\hline Gender F/M & $4 / 24$ & $1 / 11$ & $3 / 13$ \\
\hline${ }^{\dagger}$ Age, y & $11.21 \pm 3.33$ & $11.33 \pm 3.63$ & $11.63 \pm 3.18$ \\
\hline${ }^{\dagger} \mathrm{BMI}\left[\mathrm{kg} / \mathrm{m}^{2}\right]$ & $17.33 \pm 3.05$ & $17.31 \pm 3.18$ & $17.62 \pm 3.09$ \\
\hline${ }^{\dagger} \mathrm{HbA}_{1 \mathrm{c}}(\%)$ & $10.66 \pm 1.67$ & $10.59 \pm 1.15$ & $11.16 \pm 1.86$ \\
\hline${ }^{\dagger} \mathrm{C}$-peptide $[\mathrm{nmol} / \mathrm{l}]$ & $0.30 \pm 0.08$ & $0.27 \pm 0.9$ & $0.32 \pm 0.08$ \\
\hline${ }^{\dagger} 25-\mathrm{OH}-\mathrm{D}[\mathrm{ng} / \mathrm{ml}]$ & $18.19 \pm 7.68$ & $13.98 \pm 3.27^{*}$ & $21.73 \pm 8.76$ \\
\hline FDKA & $12(43)$ & $12(100)$ & $16(0)$ \\
\hline${ }^{\ddagger} \mathrm{IAA}$ & $7(25)$ & $3(25)$ & $4(25)$ \\
\hline${ }_{\mathrm{G}} \mathrm{GDA}$ & $21(75)$ & $9(75)$ & $12(75)$ \\
\hline${ }^{ \pm} \mathrm{A}$ A2A & $23(82)$ & $11(92)$ & $12(75)$ \\
\hline
\end{tabular}

$\mathrm{F}$ - female; $\mathrm{M}$ - male; $\mathrm{BMI}$ - body mass index; $\mathrm{HbA}_{1 \mathrm{c}}$ - glycated haemoglobin $\mathrm{A}_{1 \mathrm{c}}$; 25-OH-D - 25-hydroxyvitamin $\mathrm{D}$; DKA — diabetic ketoacidosis; IAA - antibodies to insulin; GADA - antibodies to glutamic acid decarboxylase; IA2A - antibodies to islet antigen-2; clinical features presented as ${ }^{\dagger}$ mean \pm standard deviation; ${ }^{\ddagger}$ number of subjects (\%); ${ }^{*} p<0.01-p$ values estimated by unpaired $t$-test, severe T1D onset vs. mild T1D onset subgroup

agents: DNA methylation, histone modification and microRNA signaling, may contribute to development of autoimmune disorders [12, 13]. MicroRNAs (miRNAs) are short ( $\sim 22$ nucleotides) non-conding RNAs regulating gene expression at the post-transcriptional level. miRNAs binds to 3'UTR of target genes and repress their expression by inhibition of translation or mRNA degradation. Whereas miRNA regulation of particular target results in modest changes in genes expression, the network activity of miRNAs might induce tremendous changes in cell behaviour [14]. Variations in gene expression, influenced by miRNAs are observed in immune system, where miRNAs modulate lymphocytes development and maturation [15-17]. Therefore, deregulation of miRNAs, which are involved in peripheral and central lymphocyte fate decision might promote autoimmunity [18-21]. Last decade demonstrated extensive efforts to examine miRNAs as T1D diagnostic and prognostic markers [22].

Our previous analysis of miRNA expression profile on Affymetrix miRNA 4.1 array in new onset T1D group indicated overexpression of miR-652-5p in patients presenting severe form of autoimmune disease [23]. We observed statistically significant upregulation of miR-652-5p in patients with initial diabetic ketoacidosis (DKA) compared to healthy controls ( $p=0.04$, fold change 4.72). The patients without DKA did not present overexpression of miR-652-5p. The aim of the study was to validate by qRT-PCR the microarray experiment in enlarged group of newly diagnosed T1D patients and investigate miR-652-5p potential target genes. Expression analysis revealed upregulation of miR-652$-5 p$ in T1D group compared to non-diabetic controls $(p<0.05)$, however we did not confirm overexpression of miR-652-5p in patient with DKA. Luciferase reporter assay did not indicated $A D A R$ and MARCH5 as miR-652-5p targets.

\section{Material and methods}

\section{Study groups}

The expression analysis of miR-652-5p was conducted in 28 newly diagnosed T1D subjects (mean age \pm SD $11.21 \pm 3.3$ years) and 28 age-matched healthy donors (mean age \pm SD $10.5 \pm 4.1$ years). Patients were recruited at the Department of Pediatric Diabetes and Obesity, Poznan University of Medical Sciences. The diagnosis of diabetes was based upon the WHO criteria. Autoimmune origin of the disease was confirmed by positive serum autoantibodies to insulin (IAA) and/ /or glutamic acid decarboxylase (GADA) and/or islet antigen-2 (IA-2A). The T1D cohort was further divided into severe and moderate onset, based upon the presence or absence of diabetic ketoacidosis (DKA) at initial presentation. The subgroup with initial DKA presented with more frequent IA2A compared to milder cases of T1D (92\% vs. 75\%). This finding suggests particularly intense autoimmune reaction and more vigorous beta cell destruction. In line, patients with severe T1D onset presented lower residual insulin synthesis reflected by lower fasting C-peptide, although the difference was statistically non-significant. Clinical characterization of patients is summarized in Table 1. To minimize the interference of the initial metabolic disorder in miRNA expression, PBMCs were collected from fully rehydrated patients with normalized ketonaemia and well-controlled glycaemia, 10 days after introduction of 
insulin therapy. Control samples with negative personal and family history of autoimmunity and no clinical signs of autoimmune disorders were obtained from an outpatient pediatric practice. The study was approved by the local Ethics Committee at the Poznan University of Medical Sciences (decision No 656/15) and all procedures were in accordance with the Declaration of Helsinki. Informed consent was obtained from parents/ /legal representatives of the minor patients.

\section{miRNA expression analysis}

The miR-652-5p expression was assessed by quantitative reverse-transcription PCR (qRT-PCR). $10 \mathrm{ng}$ of total RNA isolated from PBMCs was used in RT reaction performed with TaqMan Advance miRNA cDNA Synthesis Kit (Applied Biosystem, Thermo Fisher Scientific, US) according to the manufacturer's protocol. qRT-PCR was performed using $5 \mu \mathrm{l}$ of diluted 1:10 cDNA samples, TaqMan Fast Advance Master Mix (Applied Biosystem, CA, US) and TaqMan Advance microRNA assay (Applied Biosystem, CA, US) in a total volume of $20 \mu \mathrm{l}$. PCR reactions were run on BioRad CFX96 Real Time PCR instrument (BioRad Laboratories, CA, US). The thermal cycling conditions were an initial uracil- $\mathrm{N}$-glycosylase activation for 2 minutes at $50^{\circ} \mathrm{C}$, polymerase activation for 20 seconds at $95^{\circ}$, followed by 40 cycles of 3 seconds at $95^{\circ}$ and 30 seconds at $60^{\circ} \mathrm{C}$. All reactions were run in triplicate. RNU6 small nuclear RNA was quantified as control to normalize the differences in RNA levels. The TaqMan Advance assay 479132_mir and NR_002752.2 was used to determined the expression of miR-652-5p and RNU6, respectively. Mean cycle threshold $(\mathrm{Ct})$ values were estimated with BioRad CFX Manager 3.1 software. Relative expression levels were calculated using the $2^{-\Delta C t}$ formula.

\section{miRNA target gene prediction}

Predicted target genes for miR-652-5p were obtained from miRWalk2.0 (available at http://www.umm. uni-heidelberg.de/apps/zmf/mirwalk). Subsequently the miRNA target genes were verified with regard to their potential role in immune response and T1D pathogenesis. InnateDB (http://www.innatedb.com) and Gene Entrez database with annotation "T1D" were screened. Using the Venn's diagram (Venny 2.1.0, http://bioinfogp.cnb.csic.es/tools/venny) we identified 22 immune response genes. For further investigation 2 autoimmune related genes: Adenosine Deaminase, RNA Specific (ADAR) [24] and Membrane Associated Ring-CH Finger Protein 5 (MARCH5) [25] were selected. The sequences of miRNA bindings sites in 3'UTR of target genes were verified by PITA-Segal lab software (https://genie.weizmann.ac.il).

\section{Reporter constructs}

Binding of $A D A R$ and MARCH5 by miR-652-5p was tested by luciferase reporter assay. The 3'UTRs of selected genes were obtained from the University of California Santa Cruz genome browser on human genome hg38 assembly. $1195 \mathrm{bp}$ and $1031 \mathrm{bp}$ fragments of $A D A R$ and MARCH5 3'UTRs, respectively, were amplified from DNA of Jurkat E6.1 cell line (ATCC ${ }^{\circledR}, \mathrm{VA}$, US) purified with Gentra Puregene Blood kit (Qiagen Sciences, US). $70 \mathrm{ng}$ of DNA was used as a template in $50 \mu \mathrm{l}$ PCR reaction with $1 \mathrm{U}$ Phusion Hot Start II DNA Polymerase (Thermo Scientific, CA, US), 1X Phusion GC buffer, $200 \mu \mathrm{M}$ dNTP, $500 \mathrm{nM}$ forward and reverse primer. Sgfl restriction site was introduced into forward primer. Purified PCR products were cloned into the pGEM-Teasy vector (Promega, WI, US). Subsequently, the inserts were cleaved out using Sgfl and Notl restriction endonuclease (New England BioLabs, MA, US) and subcloned into psiCHECK2 vector (Promega, WI, US) downstream the Renilla luciferase (RL) reporter gene. The vectors' sequence was confirmed by sequencing.

\section{Luciferase assay}

Luciferase reporter assay was performed using Promega-Dual Luciferase Reporter Assay System (Promega, WI, US). $0.25 \times 10^{6}$ Jurkat cells were co-transfected with $1 \mu \mathrm{g}$ of psiCHECK2-3'UTR vector and $50 \mathrm{pmol}$ of miR-652-5p mimic or negative control \#1 (both Ambion, CA, US) using Neon Transfection System and parameters $1600 \mathrm{~V}, 10 \mathrm{~ms}, 3$ pulses. Transfected cells were dispensed into $500 \mu$ l of RPMI 1640 medium with $10 \%$ FBS, $0.25 \%$ glucose, $10 \mathrm{mM}$ HEPES, $1 \mathrm{mM}$ sodium pyruvate and incubated in $37^{\circ} \mathrm{C} / 5 \% \mathrm{CO}_{2}$. Transfections were performed in three independent experiments in triplicate. Cells lysates were made $24 \mathrm{~h}$ post transfection. Renilla and Firefly luciferase activity was measured in duplicate for each transfection on GloMax ${ }^{\circledR} 96$ Microplate Luminometer (Promega, WI, US). The expression of Renilla luciferase were normalized to secondary reporter gene Firefly luciferase. The RL/FL luciferase ratios were compared to negative control (set at 1.00). Significance was estimated with unpaired $t$-test.

\section{Statistical analysis}

Statistical analysis was performed using GraphPad Prism 5 (GraphPad Software Inc., CA, US). D'Agostino and Pearson omnibus normality test was applied to estimate if the values come from a Gaussian distribution. Data normally distributed are presented as mean \pm SD. To compare variance within each group the $F$ test was used. Statistical significance of the differences between means was determined with the unpaired $t$-test with Welch's correction. Data without Gaussian distribution 


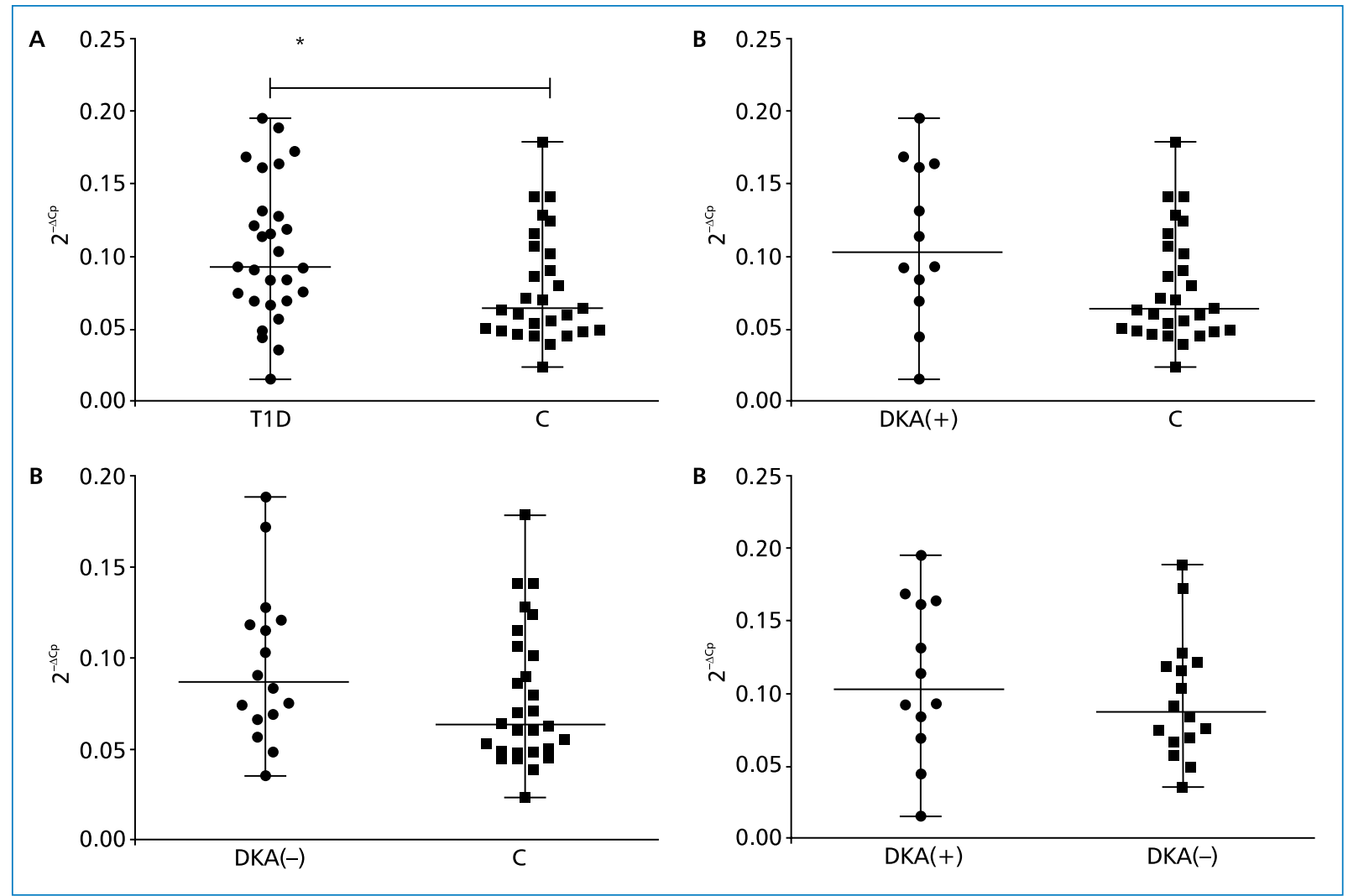

Figure 1. miR-652-5p expression analysis in T1D patients. A. miR-652-5p is significantly differentially expressed between new onset T1D and controls ( ${ }^{*} p<0.05$ ); B. miR-652-5p expression level in T1D group stratified according to the presence of initial diabetic ketoacidosis (DKA). The patients with diabetic ketoacidosis did not present statistically significant differences in miR$-652-5 p$ expression level compared to controls as well as patients without DKA $(p>0.05)$. miR-652-5p did not show significant differences between DKA(+) and DKA(-) groups. Horizontal lines indicate median with range; asterisks indicate significance, with $\mathrm{P}$ values estimated by Mann-Whitney test. T1D - type 1 diabetes patients; $\mathrm{C}$ - controls; $\mathrm{DKA}(+)$ - cohort of patients with initial diabetic ketoacidosis, DKA(-) - cohort of patients without initial diabetic ketoacidosis

are presented as median with interquartile range. Statistical significance of the differences between medians was estimated with the Mann-Whitney test. $P$ values $<0.05$ was considered statistically significant.

\section{Results}

The expression analysis by qRT-PCR revealed statistically significant upregulation of miR-652-5p in new onset type 1 diabetes patients compared to non diabetic controls (fold change 1.45, $p=0.035$ ) (Figure 1). Stratification patients group according to the presence or absence of initial diabetic ketoacidosis did not indicate the significant differences in miR-652-5p expression level compared to healthy controls $(p>0.05)$. The fold change of miR-652-5p expression reached 1.62 and 1.37 in patients with serve and mild onset of autoimmune disease, respectively, compared to controls. Furthermore, the expression of miR-652-5p was not statistically significant increased in patients with DKA versus group with mild onset of autoimmune disease ( $p>0.05$, fold change 1.18) (Figure 1).

\section{$A D A R$ and MARCH5 are not targeted by miR-652-5p}

In silico analysis was performed to indicate target genes of miR-652-5p with potential role in autoimmunity. In the cluster of 22 immune response genes we pinpointed $A D A R$ and MARCH5 for further investigation. 3'UTRs of these genes were cloned into psiCHECK2 luciferase vector.

Co-transfection of psiCHECK2-ADAR or psiCHECK2$M A R C H 5$ and miR-652-5p mimic into Jurkat cells did not indicate significant differences in relative luciferase activity compared to negative control ( $p>0.05$, Figure 2 ).

\section{Discussion}

The global incidence of T1D has been increasing by 2-3\% per year, with the most rapid increment occur- 


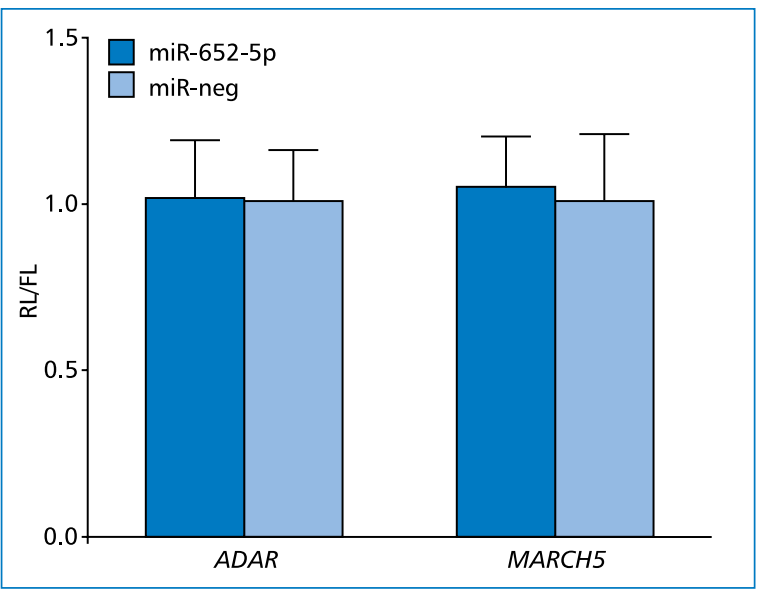

Figure 2. Luciferase reporter assay did not indicate $A D A R$ and MARCH5 as targets of miR-652-5p. Jurkat cells were cotransfected with luciferase 3'UTR reporter vectors containing $3^{\prime} U T R s$ fragment of $A D A R$ and MARCH5 genes in the presence of miR-652-5p mimic or negative control. Average Renilla/ /Firefly luciferase ratio (RL/FL) from three independent experiments is presented $\pm S D$, values for the negative control were set to 1.00 ( $t$ test $p>0.05$ )

ring among the youngest children ( $<5$ years of age). A recent decrease in high-risk HLA genetic contribution in new-onset cases versus overall increase in T1D incidence might suggest the changing environmental pressure on disease development and involvement of other genes and epigenetic factors [26].

Last decade demonstrate extensive efforts in understanding the role of specific miRNAs in pathogenesis of T1D, which pinpoint miRNAs as diagnostic and prognostic biomarkers of autoimmune disorders.

In current study we observed overexpression of miR-652-5p in newly diagnosed T1D patients compared to healthy controls. T1D patients presented ongoing autoimmune process indicated by positive serum autoantibodies to insulin (IAA) and/or glutamic acid decarboxylase (GADA) and/or islet antigen-2 (IA-2A). Expression analysis did not display significantly higher miR-652-5p expression level in patients with severe form of T1D compared to control group. The observation stays in opposite to our previous microarray experiment [23]. The discrepancy in expression level between microarray and validation analysis might be partially explained by the relative small sample size of microarray cohort (patients with DKA, $n=7$ ). Patients with initial diabetic ketoacidosis presented trend of miR-652-5p upregulation compared to patients without DKA, although the differences did not reach statistical significance. miR-652-5p has not been previously reported in the context of autoimmune disease and it is likely to be a potential biomarker of new onset T1D. Limitation of current investigation is small sample size, therefore further extension of study group is required to affirm miR-652-5p as epigenetic T1D risk factor.

Furthermore, studies concerning miRNAs expression analysis in PBMCs and serum of adult type 1 diabetes patients, revealed other miRNAs that might be associated with autoimmunity [27-29]. miR-326 was found to be upregulated in PBMCs from autoantibody-positive T1D patients [29]. In mice, overexpression of miR-326 during experimental autoimmune encephalomyelitis leads to increased Th17 cell differentiation [30]. In contrast, Salas-Perez et al. observed downregulation of miR-21a and miR-93 in PBMC from T1D patients [31]. Interestingly, the in silico analysis of their target genes revealed pro-apoptotic and pro-inflammatory Caspase 8, Caspase 7 and IL-8 for miR-93 as well as Fas ligand, MTPN, APAF1, IL-12A, IL-22 and IL-1B genes for miR-21.

Recent studies demonstrated miRNAs as potential tool for disease staging. It was indicated that decreased miR-146 expression in PBMCs correlate with ongoing islet autoimmunity and high serum GADA titers [28]. In addition, dysregulation of miR-125b-5p, miR-365a-3p, miR-5190 and miR-770-5p was associated with hyperglycemia in patients with T1D and might contribute to the development of diabetes complications [32]. Pathway analysis revealed that 50 Kyoto Encyclopedia of Genes and Genomes pathways were significantly enriched by genes targeted by these four miRNAs [32].

An important strategy for understanding the role of miRNAs in pathogenesis of autoimmune disease is to identify their target genes. We are particularly interested in the potential mechanism linking innate immunity and development of type 1 diabetes. Therefore, we validated $A D A R$ and MARCH5 as potential miR-652-5p targets by luciferase reporter assay. The mutations in ADAR were indicated to cause the autoimmune disorder Aicardi-Goutières syndrome [24, 33, 34]. As in Adar1-null mice, the human disease state is associated with upregulation of interferonstimulated genes [33]. The study of Dominguez-Gutierrez et al. showed overexpression of $A D A R$ in Systemic Lupus Erythematosus (SLE) patients [35]. In addition, decreased MARCH5 protein might account for the constant Mitochondrial Antiviral-Signaling Protein aggregations and abnormalities of type I interferon levels in SLE patients [25]. Current study did not indicate $A D A R$ and $M A R C H 5$ as miR-652-5p targets in luciferase reporter assay. Therefore, we hypothesize that miR-652-5p is unlikely to regulate $A D A R$ and MARCH5 expression.

In conclusion, current study revealed miR-652-5p as potential marker of new onset type 1 diabetes. Investigation miRNAs in context of autoimmunity is important issue as improved biomarkers are required for the detection of developing type 1 diabetes, prior to critical loss of pancreatic beta cells. 


\section{Conflicts of interests}

All authors declare that there are no conflicts of interest associate with this publication.

\section{Acknowledgments}

The study was supported by Polish Diabetes Association (Polskie Towarzystwo Diabetologiczne), Artur Czyzyk Research Grant 2015.

\author{
Disclosure statement: The authors have nothing \\ to disclose
}

\section{REFERENCE}

1. Bluestone JA, Herold K, Eisenbarth G. Genetics, pathogenesis and clinical interventions in type 1 diabetes. Nature. 2010; 464(7293): 1293-1300, indexed in Pubmed: 20432533.

2. Davies JL, Kawaguchi Y, Bennett ST, et al. A genome-wide search for human type 1 diabetes susceptibility genes. Nature. 1994; 371(6493): 130-136, doi: 10.1038/371130a0, indexed in Pubmed: 8072542.

3. Aly TA, Ide A, Jahromi MM, et al. Extreme genetic risk for type 1A diabetes. Proc Natl Acad Sci U S A. 2006; 103(38): 14074-14079, doi: 10.1073/pnas.0606349103, indexed in Pubmed: 16966600.

4. Ueda H, Howson JMM, Esposito L, et al. Association of the T-cell regulatory gene CTLA4 with susceptibility to autoimmune disease. Nature. 2003; 423(6939): 506-511, doi: 10.1038/nature01621, indexed in Pubmed: 12724780.

5. Bottini N, Musumeci L, Alonso A, et al. A functional variant of lymphoid tyrosine phosphatase is associated with type I diabetes. Nat Genet. 2004; 36(4): 337-338, doi: 10.1038/ng1323, indexed in Pubmed: 15004560.

6. Smyth DJ, Cooper JD, Bailey R, et al. A genome-wide association study of nonsynonymous SNPs identifies a type 1 diabetes locus in the interferon-induced helicase (IFIH1) region. Nat Genet. 2006; 38(6): 617-619, doi: 10.1038/ng1800, indexed in Pubmed: 16699517.

7. Lowe CE, Cooper JD, Brusko T, et al. Large-scale genetic fine mapping and genotype-phenotype associations implicate polymorphism in the IL2RA region in type 1 diabetes. Nat Genet. 2007; 39(9): 1074-1082, doi: 10.1038/ng2102, indexed in Pubmed: 17676041.

8. Vaarala $\mathrm{O}$, Klemetti $\mathrm{P}$, Juhela $\mathrm{S}$, et al. Effect of coincident enterovirus infection and cows' milk exposure on immunisation to insulin in early infancy. Diabetologia. 2002; 45(4): 531-534, doi: 10.1007/s00125-002-0787-8, indexed in Pubmed: 12032629.

9. Wasmuth HE, Kolb H. Cow's milk and immune-mediated diabetes. Proc Nutr Soc. 2000; 59(4): 573-579, indexed in Pubmed: 11115792.

10. Wen Li, Ley RE, Volchkov PYu, et al. Innate immunity and intestinal microbiota in the development of Type 1 diabetes. Nature. 2008; 455(7216): 1109-1113, doi: 10.1038/nature07336, indexed in Pubmed: 18806780

11. Weintrob N, Sprecher E, Israel S, et al. Type 1 diabetes environmental factors and correspondence analysis of HLA class II genes in the Yemenite Jewish community in Israel. Diabetes Care. 2001; 24(4): 650-653, indexed in Pubmed: 11315825.

12. Brown CC, Wedderburn LR. Genetics: Mapping autoimmune disease epigenetics: what's on the horizon? Nat Rev Rheumatol. 2015; 11(3): 131-132, doi: 10.1038/nrrheum.2014.210, indexed in Pubmed: 25512011.

13. Jeffries MA, Sawalha AH. Autoimmune disease in the epigenetic era: how has epigenetics changed our understanding of disease and how can we expect the field to evolve? Expert Rev Clin Immunol. 2015; 11(1): 45-58, doi: 10.1586/1744666X.2015.994507, indexed in Pubmed: 25534978.
14. Simpson $\sqcup$, Ansel KM. MicroRNA regulation of lymphocyte tolerance and autoimmunity. J Clin Invest. 2015; 125(6): 2242-2249, doi: 10.1172/JCl78090, indexed in Pubmed: 26030228.

15. Baumjohann D, Ansel KM. MicroRNA-mediated regulation of $T$ helper cell differentiation and plasticity. Nat Rev Immunol. 2013; 13(9): 666-678, doi: 10.1038/nri3494, indexed in Pubmed: 23907446.

16. O'Connell RM, Rao DS, Baltimore D. microRNA regulation of inflammatory responses. Annu Rev Immunol. 2012; 30: 295-312, doi: 10.1146/annurev-immunol-020711-075013, indexed in Pubmed: 22224773.

17. Xiao C, Rajewsky K. MicroRNA control in the immune system: basic principles. Cell. 2009; 136(1): 26-36, doi: 10.1016/j. cell.2008.12.027, indexed in Pubmed: 19135886.

18. Ventura A, Young AG, Winslow MM, et al. Targeted deletion reveals essential and overlapping functions of the miR-17 through 92 family of miRNA clusters. Cell. 2008; 132(5): 875-886, doi: 10.1016/j.cell.2008.02.019, indexed in Pubmed: 18329372.

19. Xiao C, Srinivasan L, Calado DP, et al. Lymphoproliferative disease and autoimmunity in mice with increased miR-17-92 expression in lymphocytes. Nat Immunol. 2008; 9(4): 405-414, doi: 10.1038/ /ni1575, indexed in Pubmed: 18327259.

20. Li QJ, Chau J, Ebert PJR, et al. miR-181a is an intrinsic modulator of T cell sensitivity and selection. Cell. 2007; 129(1): 147-161, doi: 10.1016/j.cell.2007.03.008, indexed in Pubmed: 17382377.

21. Kang SG, Liu WH, Lu P, et al. MicroRNAs of the miR-17 92 family are critical regulators of $\mathrm{T}(\mathrm{FH})$ differentiation. Nat Immunol. 2013; 14(8): 849-857, doi: 10.1038/ni.2648, indexed in Pubmed: 23812097.

22. Zheng Y, Wang Z, Zhou Z. miRNAs: novel regulators of autoimmunity-mediated pancreatic $\beta$-cell destruction in type 1 diabetes. Cell Mol Immunol. 2017; 14(6): 488-496, doi: 10.1038/ /cmi.2017.7, indexed in Pubmed: 28317889.

23. Zurawek M, Dzikiewicz-Krawczyk A, Izykowska K, et al. miR487a-3p upregulated in type 1 diabetes targets CTLA4 and FOXO3. Diabetes Res Clin Pract. 2018 [Epub ahead of print]; 142: 146-153, doi: 10.1016/j.diabres.2018.05.044, indexed in Pubmed: 29859273.

24. Rice GI, Kasher PR, Forte GMA, et al. Mutations in ADAR1 cause Aicardi-Goutières syndrome associated with a type I interferon signature. Nat Genet. 2012; 44(11): 1243-1248, doi: 10.1038/ /ng.2414, indexed in Pubmed: 23001123.

25. Shao WH, Shu DH, Zhen Y, et al. Prion-like Aggregation of Mitochondrial Antiviral Signaling Protein in Lupus Patients Is Associated With Increased Levels of Type I Interferon. Arthritis Rheumatol. 2016; 68(11): 2697-2707, doi: 10.1002/art.39733, indexed in Pubmed: 27110677.

26. Fourlanos S, Varney MD, Tait BD, et al. The rising incidence of type 1 diabetes is accounted for by cases with lower-risk human leukocyte antigen genotypes. Diabetes Care. 2008; 31(8): 1546-1549, doi: 10.2337/dc08-0239, indexed in Pubmed: 18487476.

27. Hezova R, Slaby O, Faltejskova P, et al. microRNA-342, microRNA-191 and microRNA-510 are differentially expressed in T regulatory cells of type 1 diabetic patients. Cell Immunol. 2010; 260(2): 70-74, doi: 10.1016/j.cellimm.2009.10.012, indexed in Pubmed: 19954774.

28. Yang $M$, Ye $L$, Wang $B$, et al. Decreased miR-146 expression in peripheral blood mononuclear cells is correlated with ongoing islet autoimmunity in type 1 diabetes patients $1 \mathrm{miR}-146$. J Diabetes. 2015; 7(2): 158-165, doi: 10.1111/1753-0407.12163, indexed in Pubmed: 24796653.

29. Sebastiani G, Grieco FA, Spagnuolo I, et al. Increased expression of microRNA miR-326 in type 1 diabetic patients with ongoing islet autoimmunity. Diabetes Metab Res Rev. 2011; 27(8): 862-866, doi: 10.1002/dmrr.1262, indexed in Pubmed: 22069274.

30. Du C, Liu C, Kang J, et al. MicroRNA miR-326 regulates TH-17 differentiation and is associated with the pathogenesis of multiple sclerosis. Nat Immunol. 2009; 10(12): 1252-1259, doi: 10.1038/ /ni.1798, indexed in Pubmed: 19838199. 
31. Salas-Pérez F, Codner $E$, Valencia $E$, et al. MicroRNAs miR-21 $a$ and miR-93 are down regulated in peripheral blood mononuclear cells (PBMCs) from patients with type 1 diabetes. Immunobiology. 2013; 218(5): 733-737, doi: 10.1016/j.imbio.2012.08.276, indexed in Pubmed: 22999472.

32. Satake E, Pezzolesi MG, Md Dom Zl, et al. Circulating miRNA Profiles Associated With Hyperglycemia in Patients With Type 1 Diabetes. Diabetes. 2018; 67(5): 1013-1023, doi: 10.2337/db171207, indexed in Pubmed: 29453204.

33. Rice GI, Forte GMA, Szynkiewicz M, et al. Assessment of interferon-related biomarkers in Aicardi-Goutières syndrome associated with mutations in TREX1, RNASEH2A, RNASEH2B, RNASEH2C,
SAMHD1, and ADAR: a case-control study. Lancet Neurol. 2013; 12(12): 1159-1169, doi: 10.1016/S1474-4422(13)70258-8, indexed in Pubmed: 24183309.

34. Crow YJ, Chase DS, Lowenstein Schmidt J, et al. Human Disease Phenotypes Associated With Mutations in TREX1. Journal of Clinical Immunology. 2015; 35(3): 296-312, doi: 10.1002/ajmg.a.36887.

35. Dominguez-Gutierrez PR, Ceribelli A, Satoh M, et al. Elevated signal transducers and activators of transcription 1 correlates with increased $\mathrm{C}-\mathrm{C}$ motif chemokine ligand 2 and $\mathrm{C}-\mathrm{X}-\mathrm{C}$ motif chemokine 10 levels in peripheral blood of patients with systemic lupus erythematosus. Arthritis Res Ther. 2014; 16(1): R20, doi: 10.1186/ar4448, indexed in Pubmed: 24451065. 